\title{
Hormones, Brain, and Neuropsychopharmacology
}

Uriel Halbreich, M.D.

During the last decade, there has been an explosion in our knowledge of the interaction between brain, hormones and behavior. Many hormones have been shown to modulate central nervous system functions, and alterations in hormonal activity are associated with several major mental and neurological disorders. Basic and clinical studies have provided the foundation for the development of synthetic compounds acting through hormonal systems that are putatively involved in the modulation of normal and abnormal behaviors, cognition and mood. The more recent data are a further evolution from the well-documented knowledge that many psychotropic medications alter activity of various hormonal systems. Furthermore, most hormonal abnormalities are associated with mental changes and changes in hormonal milieu influence the activity of many psychotropic drugs. Gender differences in drug efficacy and side effects are also, at least partially, attributed to hormonal differences.

Conceptually, the evolvement of the field points to a shift from a simplistic unidimensional stimulus-effect approach, to a multi-dimensional, multiinteractional appreciation of the processes that are shaping mood and behaviors.

On a practical level, the multi-faceted complexity of this fascinating evolving area calls for a close interdisciplinary collaboration towards better understanding and practical implementation of knowledge in clinical practice. The quest for integration will be enhanced on several levels: a) Integration of Basic Neurosciences, Clinical Research and Clinical Practice; b) Integration and Close Collaboration Between Academia, Industry and Private Practice; and c) Emphasis on Interdisciplinary Work with Psychiatry, Neurology and Endocrinology on the Clinical Side, and Molecular, Cellular and Biobehavioral Neurosciences as well as Pharmacology, Drug Development and Drug Testing-On the Bench Side.

The conceptual multi-dimensional approach to the topic is also expressed in the administrativeorganizational structure of the First International Congress on Hormones, Brain and Neuropsychopharmacology. The Congress consists of three tracks, which might be perceived as "Congresses within a Congress": a) Clinical Neuropsychopharmacology; b) Basic Neurosciences; and c) Pharmacoendocrinology. The three scientific tracks are all structured with the focus to the future. The main questions that we hope to address are: "What new treatment modalities are being developed?" "What are the implications of recent achievements to new drug development?" "How are various aspects interacting with each other, and what shall we do to achieve interaction and integration?"

In each session, we will emphasize future directions for research and clinical implications as well as diversity, controversy and data-based predictions. Hopefully, our prediction for a stimulating, intriguing and productive Congress will be realized. 